BRE 11545

\title{
A Microcomputer-Based Image Analyzer for Quantitating Neurite Outgrowth
}

\author{
THOMAS S. FORD-HOLEVINSKI, TODD A. DAHLBERG and BERNARD W. AGRANOFF \\ Neuroscience Laboratory Building, University of Michigan, Ann Arbor, MI 48109 (U.S.A.)
}

(Accepted July 30th, 1985)

Key words: image processing - quantitation - computer — retinal explant

\begin{abstract}
An automated device that recognizes and quantitates neurite outgrowth from goldfish retinal explants is described. The system components include a video camera-inverted microscope and an Apple II microcomputer with an attached Motorola 68000 coprocessor. Values for neurite outgrowth were generated within $30 \mathrm{~s}$, required no user input other than defining the microscopic field, and correlated well with a subjective measure, the nerve growth index.
\end{abstract}

\section{INTRODUCTION}

Agents that either stimulate or block neuronal growth have been characterized by means of assay procedures that measure effects of these substances on outgrowth of neurites in culture ${ }^{3,13}$. Such methods require estimating average neurite length and the number of neurites or parameters that attempt to calculate the density of outgrowth (grid crossings, etc.). In an attempt to introduce more objectivity to such assays, Dombrowski and Kauffman ${ }^{4}$ measured the activity of isocitrate dehydrogenase in neuritic outgrowth from superior cervical ganglia. After outgrowth, the preparation was lyophilized and the central explant excised prior to enzyme measurement. A disadvantage of this procedure is that each explant can be measured only once, in contrast to repeated visual measurements made in conventional nerve growth assays. Also, the investigator must ascertain that the biochemical changes examined are indeed related to the mass of outgrowth and are unaffected by various treatments to which the cells or explants may have been subjected.

Some investigators, taking advantage of new computer technology, have attempted to automate the analysis of neurite outgrowth in order to eliminate the subjective nature of previously employed visual estimations, while preserving their non-invasive aspect. The majority of such systems have used digitizing pads on which images of individual neurites may be traced ${ }^{5}$ or on which the perimeter of outgrowth may be defined ${ }^{l}$. Alternatively, video microscope signals have been digitized and manipulated by algorithms designed to enhance neurite definition 2,5,6. Until now, these latter techniques suffered from several problems: they required significant user input, took unacceptably long periods of processing time or required the use of prohibitively expensive components. Additionally, prior attempts to automate neurite measurement did not eliminate imaged debris nor did they restore artifactual breaks in neurite continuity.

The present project describes the development of an image processor constructed of commercially available and inexpensive components which, when applied to an inverted microscope, quickly provides a completely objective measure of neurite outgrowth.

\section{MATERIALS AND METHODS}

Goldfish retinal explant culture

The procedure for maintaining retinal explants in

Correspondence: B.W. Agranoff, Neuroscience Laboratory Building, University of Michigan, 1103 East Huron, Ann Arbor. MI 48109. U.S.A. 
culture has been described elsewhere ${ }^{9,10}$. It is based on a conditioning lesion made in vivo several days prior to explantation. Retinas were cut by a McIlwain mechanical chopper (Brinkmann, Westbury, NY) into $0.5 \mathrm{~mm}$ squares and were positioned as a $3 \times 3$ matrix on $35 \mathrm{~mm}$ plastic culture dishes precoated with poly-L-lysine. They were maintained in air in a moist chamber at $21{ }^{\circ} \mathrm{C}$ and received no additions or changes of medium during the following 8 days.

\section{Nerve growth index (NGI)}

The NGI was estimated as described by Landreth and Agranoff 11 . This method involves the estimation of two growth variables: neurite density and average neurite length. Assigned neurite density values range from 0 to 4 , based on a photographic atlas of previously grown explants. A score of 4 indicates maximum density. A micrometer in the microscope eyepiece is used to measure average neurite length using a range of $0-4$ explant diameters $(0.5 \mathrm{~mm}$ units). These two values are then multiplied to obtain the NGI

Computer-assisted nerve growth index (CNGI): hardware

Retinal explants in culture were examined by pseudo-darkfield microscopy (phase contrast condenser ring with a non-phase objective) using a Leitz

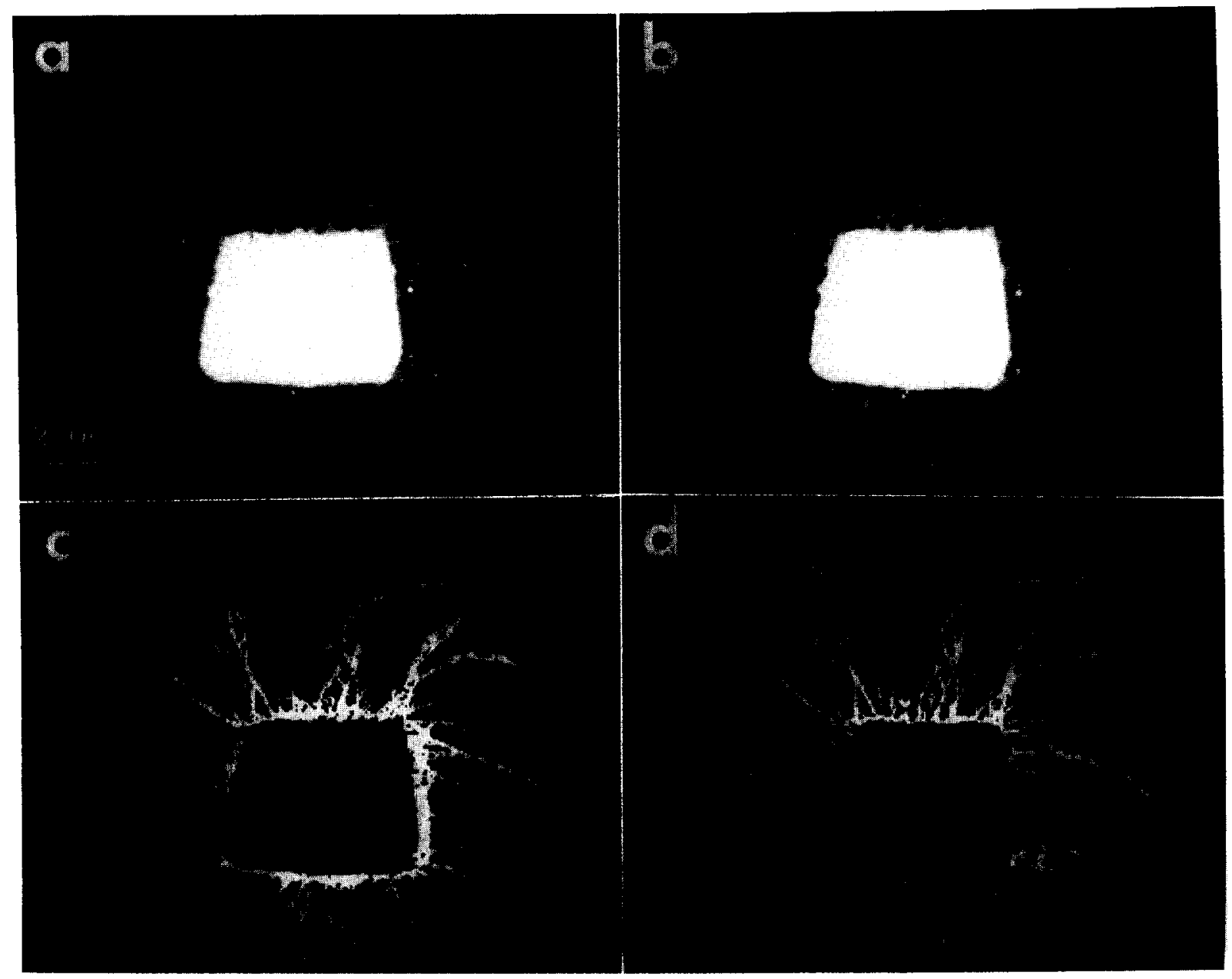

Fig. 1. Imaging a goldfish retinal explant after 6 days in culture. a: photomicrograph of explant. b: video image of explant. Note some loss of detail and increased contrast relative to photomicrograph. c: image generated when all the pixels between two intensity thresholds were lit. These intensity thresholds were set so as to maximize neurite visualization. Note the abundance of lit pixels in the central region of the screen and their lack near its edges. d: image generated when 'Float', the algorithm which alters the thresholds at each point on the basis of the surround pixel values, was used (see Methods). 
Diavert microscope and its $2.5 \times$ objective (Fig. 1a). A lensless Sony AGC-3200 black and white video camera was attached to the microscope to accept the image. The video signal was relayed to a standard video monitor to allow proper focusing and centering of the explant to be analyzed (Fig. 1b). It was also sent to a digitizing circuit (Dithertizer II; Computer Stations, Inc., St. Louis, MO) installed in an Apple II microcomputer (Apple Computer, Inc., Cupertino, CA; Fig. 2). The Dithertizer II contains an 8 bit ana$\log$ to digital converter and a 1 bit comparator which holds a value from 0 to 255 . At locations in the video image which correspond to picture elements (pixels) on the Apple II high resolution graphics screen (280 elements horizontally by 192 vertically) the analog video signal is converted into an integer value, also from 0 to 255. If the digital value for a given point on the video screen is greater than the value stored in the comparator, the corresponding pixel on the graphics screen of the Apple II is then set to white. Otherwise it is set to black. Using direct memory access this device digitizes an entire image in $1 / 60$ th of a second.

Attached to the Apple computer was a Dtack Grande coprocessor board and its Stuffer interface (Digital Acoustics, Santa Ana, CA). The board contains a Motorola 68000 microprocessor, running at an effective rate of $10 \mathrm{MHz}$, and 128 Kbytes of memory, expandable to 1 Mbyte.

\section{CNGI: software routines}

Capture is the routine which acquires 256 one bit images and combines them in the 68000 board to form a single 8 bit ( 1 byte) image. It starts by setting

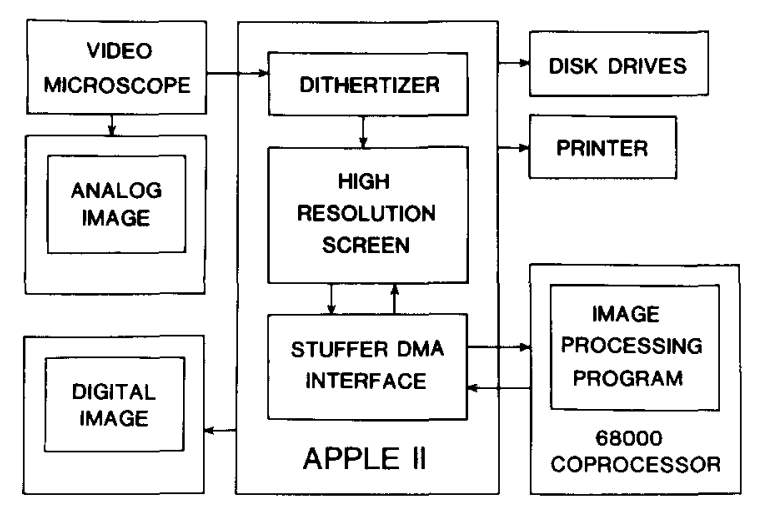

Fig. 2. Schematic of Apple II image processor hardware. the intensity threshold in the 1 bit comparator to 255 , the 'brightest' setting available. At this setting only the extremely bright regions of the image are lit on the graphics screen. In practice, however, few pixels are on at this intensity. The binary image generated on the Apple graphics screen by the Dithertizer II is sent to the 68000 board, taking $70 \mathrm{~ms}$ after the coprocessor has signalled that it is ready for an image. Once in the coprocessor, the 68000 assembly language program subtracts the new image from the image obtained at the previous intensity threshold to determine which pixels are uniquely lit at the current intensity. The intensity value for that scan is then stored in a 1 byte location which corresponds to the lit pixel's location on the graphics screen. The 68000 board then signals its readiness to accept another image and the storage process is repeated until the intensity value is 0 . In this way a 1 byte ( 8 bit) value represents each pixel on the graphics screen. Once this process is complete (approximately $12 \mathrm{~s}$ ), the operator can center and focus the next explant to be examined.

Average is the 68000 assembly language routine which removes low frequency noise from the image by replacing each pixel with the average of the $3 \times 3$ matrix of pixels which surrounds it. Each pixel contributes equally to this average, but only pixels with an intensity value within 3 units of the central pixel are included. This cut-off maintains edges, so the imaged neurites are thus a pattern of edges on a mottled background. This routine takes approximately $4 \mathrm{~s}$.

Replace is the routine which eliminates the image of the explant body. First the maximum pixel value in the image is established. Then all pixels with intensities within 18 units of this maximum are set to the value 255 . Once raised to this value, these pixels are effectively removed from further calculations. Since the explant body is much brighter than the surrounding neurites, this will set its pixel values to 255 without altering any neurite values. This routine takes less than $2 \mathrm{~s}$.

Float is the routine which produces a binary representation of the image which then can be subjected to pattern recognition. We found we could not obtain a reasonable image of our neurites by simply turning on all the pixels between two thresholds (see Fig. 1c). This is in part due to uneven microscope illumination 
and video tube sensitivity, but is mainly due to a corona of diffuse illumination that occurs around the explant body. As a result, neurites near the explant body are on a brightly lit background while those near the edges of the field are dimly lit. To overcome these effects we devised an algorithm that would determine the best lower threshold at each point in the image using the values of those pixels in the $3 \times 3$ matrix which surrounds it. In this instance, the cut-off range is not used. After the average of the surround is calculated, a constant (1) is added to raise it above the finer background noise caused by plastic dish irregularities, the media, small particulates, etc. This value is the lower threshold. Finally, a constant (64) is added to this value to give the upper threshold. If the pixel in question falls between these thresholds, then it is set to white on the graphics screen. This gives an image that has the appearance of being evenly lit and maximizes the visualization of fine details, such as neurites. This routine takes approximately $4 \mathrm{~s}$.

Recognize is the final processing step. This routine uses pattern recognition algorithms to eliminate debris and fill in small missing portions of neurites. It then determines how many pixels on the screen are lit. Starting at the upper left corner and continuing to the lower right corner, pattern recognition is performed at each lit pixel as follows: 33 predefined patterns of pixels, designed to approximate polar vectors radiating from the lit test pixel (see inset, Fig. 4), are sequentially examined for lit pixels (Fig. 4). These search patterns are each 8 pixels in length, including the test pixel. If 3 consecutive unlit pixels are encountered, that search pattern is terminated as invalid and the next one is examined. It is possible that none of the search patterns will be valid, in which case the test pixel will be identified as debris and ignored. The pattern recognition procedure will then be performed on the next lit pixel. If, however, valid 'vectors' are found in this primary search, a similar process is performed at the terminus of the pattern with the greatest number of lit pixels. Here 13 additional search patterns are examined, one in the same direction as the identified primary pattern, and 6 to either side (Fig. 4). The gap criterion must also be met for these secondary search patterns. If valid secondary search patterns are found, then both the best primary and secondary search patterns are plotted on a duplicate high resolution graphics screen which will eventually represent the neuritic outgrowth. The small gaps that may have been present in the final search patterns are now plotted as lit pixels, filling in

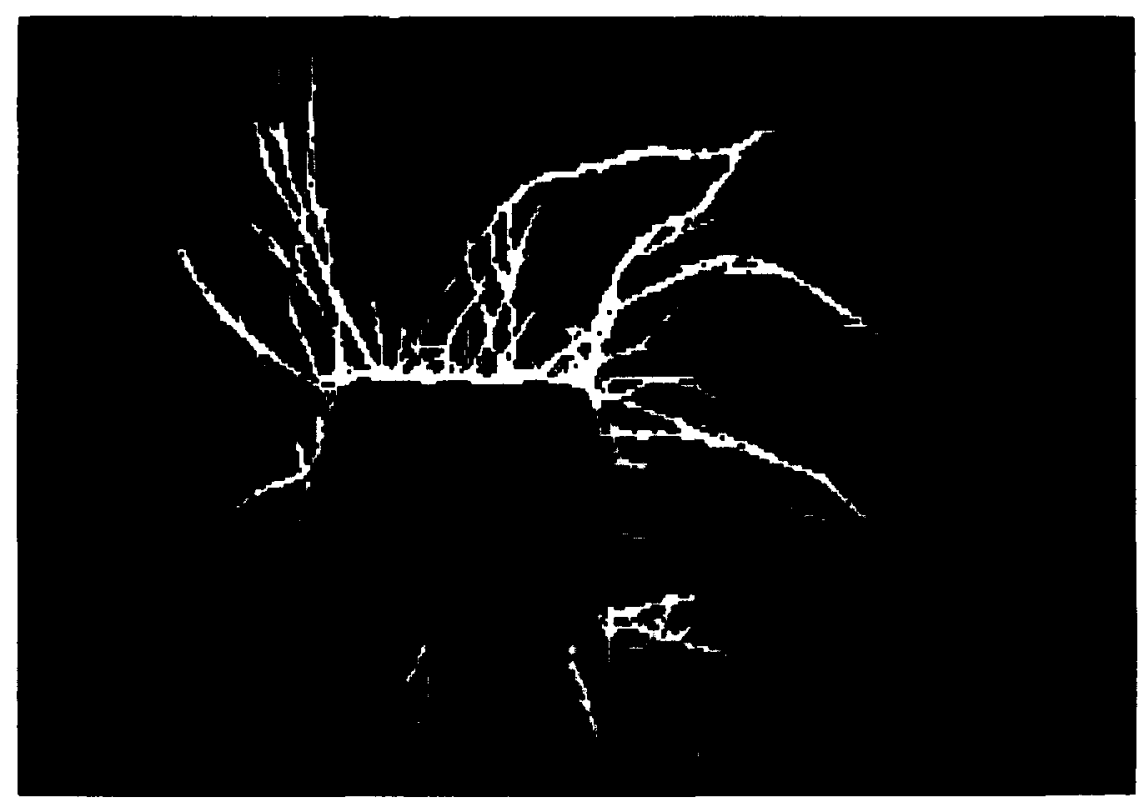

Fig. 3. Final image of explant culture, produced when 'Recognize', the pattern recognition algorithm, was used on the image in Fig. 1d (see Methods). Note loss of debris and continuity of neurite images. 

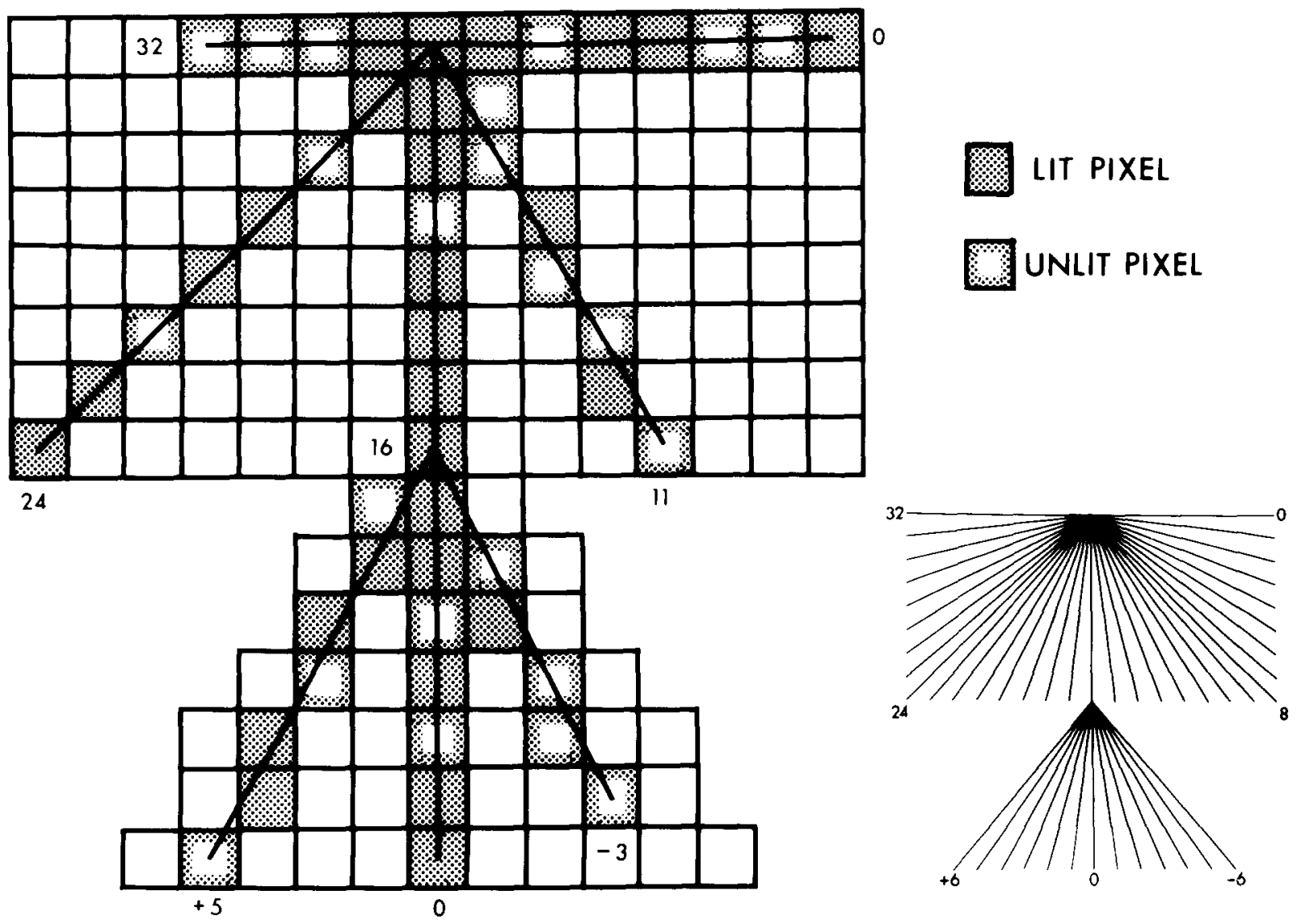

Fig. 4. Search patterns used by the pattern recognition routine. Each lit pixel first undergoes a $180^{\circ}$ primary scan which examines 33 predefined patterns approximating polar vectors radiating from the test pixel (inset). For clarity, only the pixels in 'vectors' numbered $0,11,16,24$ and 32 are shown. Encountering a gap of 3 consecutive pixels renders a vector invalid (e.g. number 32 ). A $67.5^{\circ}$ secondary search is conducted from the terminus of the primary search pattern with the most lit pixels. This includes the search pattern with the orientation of the chosen primary search pattern, as well as 6 search patterns to either side of that (inset). Only secondary 'vectors' 0 , -3 and +5 are shown for clarity. Secondary vector number -3 was terminated when it failed to meet the gap criterion. In this instance primary search pattern no. 16 and secondary search pattern no. 0 would be plotted (without gaps) on a second graphics page as valid portions of neurite (see Methods for complete details).

discontinuities in the string of pixels which has been defined to constitute a neurite image. If no valid secondary search patterns are found, then the original test pixel is defined as debris. This process is repeated until all lit pixels on the debris-filled binary image have been tested. The number of lit pixels on the final, cleaned image is used as the measure of neuritic outgrowth. Depending on the number of pixels lit in the original debris-filled image, this routine takes $5-10 \mathrm{~s}$.

\section{RESULTS}

When 29 explants were quantitated at 4, 6 and 8 days in culture by two independent observers using the NGI, and the averages of these 87 values were then compared to those obtained with the computerized system (Fig. 5a), the correlation coefficient (0.94) was greater than that obtained when the two sets of NGI values were compared to one another (0.88). This indicated that the CNGI was at least as quantitative as the NGI, in addition to being completely objective. We then plotted the percent deviation of the CNGI values from the best linear fit to the data shown in Fig. 5a against the actual NGI values (Fig. 5b) to determine whether the CNGI was systematically discrepant. The deviations were equally spaced both positively and negatively (as indicated 

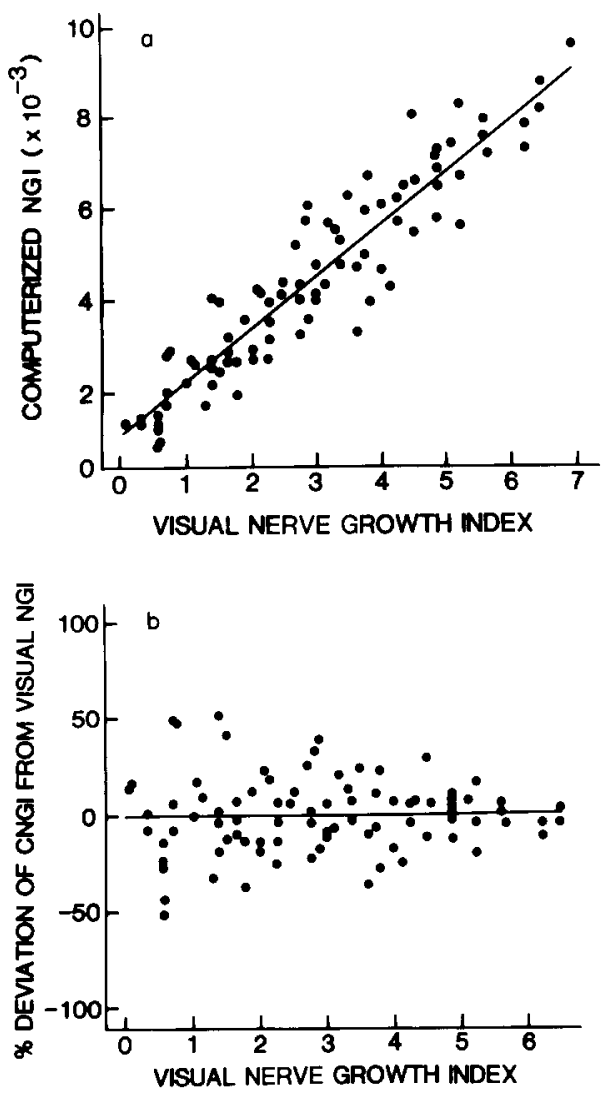

Fig. 5. A comparison of the CNGI and the NGI. a: plot of the computerized NGI values against the average of two visual NGI readings for each explant over 3 days in culture showing the best linear fit. Correlation coefficient $=0.94(n=87)$. b: deviation of $\mathrm{CNGI}$ values from the linear best fit to the data shown in Fig. 5a, determined as [100× (actual CNGI-calculated $\mathrm{CNGI}$ /calculated $\mathrm{CNGI}$ ] and plotted against the NGI values. The best linear fit to this data is also shown.

by the best linear fit which has both a zero slope and Y-intercept), but were greater at the lower end of the scale. This may be due to the system's occasional inability to distinguish edges of the explant from neurites (see Figs. 1 and 3). As the outgrowth increases these edges contribute fractionally less to the total number of lit pixels and as a result, accuracy increases.

The values used in Fig. 5a were also plotted as growth curves to see whether the methods compared at various times of growth in vitro. As shown in Fig. 6 , the curves for the two NGIs and the CNGI were very similar in appearance with no significant differences as a function of time (as analyzed by both ANOVA and Student's $t$-test). Minor differences ob-



Fig. 6. Growth curves of goldfish explants over 8 days in culture plotted as the percent of mean for each method on day 8 . Computerized nerve growth index ( $\boldsymbol{0}$ ) and visual nerve growth index viewer no. $1(\boldsymbol{O})$ and viewer no. $2(\boldsymbol{\Delta})$. Error bars indicate standard deviations $(\mathrm{n}=20$ for each time point $)$.

served in the CNGI and NGI curves may be related to differences in light intensities or condenser settings, since we did not standardize these parameters from day to day. It may prove desirable to use a preserved specimen to calibrate the mechano-optical portion of the system prior to use.

As a test of system precision, an explant was scanned repetitively without movement between scans. We found that the coefficient of variation of the growth values obtained was $2.2 \%(\mathrm{n}=25)$ and that this increased to only $2.5 \%(\mathrm{n}=10)$ if the explant was repositioned by both translation and rotation.

Occasional cultures, so congested with cellular debris or scratches on the dish that quantitation by the NGI is difficult, also present problems for CNGI determination. In these cases no attempt is made at computerized quantitation, and since this decision is made by the observer, a degree of subjectivity enters into this method as well. We also found that many explants outgrow the magnification limits of the system after 1-2 weeks in culture. In most cases this does not present a problem as we are usually interested in initial rates of outgrowth, and growth in most cultures begins to level off or regress by the end of 2 weeks. When we attempted to overcome this problem by using a $1 \times$ objective, the accuracy of the system was greatly reduced, as indicated by a correlation coefficient of $0.56(n=27)$ obtained when $C N G I$ values were compared to NGI values. When the effective 
resolution was increased through the use of a $4 \times$ objective, much more detailed digital images were produced, but most explants outgrew the field of this objective in a few days.

\section{DISCUSSION}

Investigators using the goldfish retinal explant system and other neuronal cell cultures have quantitated the growth of neurites primarily by visual estimates. Since these neurites are less than $1 \mu \mathrm{m}$ in diameter and are attached either to the substratum or to other neurites, the outgrowth may be considered to be constant in depth and regarded as two-dimensional. The present automated system approximates some of the recognition operations performed by visual inspection, taking advantage of the linear nature of the neurites to distinguish them from globular debris and to fill in small discontinuities in their video images.

At the magnification normally used $(2.5 \times$ objective), the resolution of our system is approximately 9 $\mu \mathrm{m}$ horizontally and 10 vertically. Although the neurites are much smaller than this, they are visible to the eye and the video camera because they scatter light by the pseudo-darkfield illumination used and because they commonly grow out in fascicles, or bundles. As a result, the $280 \times 192$ pixel resolution of the Apple II was sufficient to record the outgrowth of neurites from our cultures with reasonable accuracy. It is probable that using commercially available image processing systems with higher resolutions (e.g. $512 \times 512$ pixels or more), even more accurate estimates of growth could be obtained. The limitation of resolution in system accuracy was very ob-

\section{REFERENCES}

1 Argiro, V. and Johnson, M.I., Patterns and kinetics of neurite extension from sympathetic neurons in culture are age dependent, J. Neurosci., 2 (1982) 503-512.

2 Argiro, V., Bunge, M.B. and Johnson, M.I., Correlation between growth form and movement and their dependence on neuronal age, J. Neurosci., 4 (1984) 3051-3062.

3 Collins, F. and Dawson, A., An effect of nerve growth factor on parasympathetic neurite outgrowth, Proc. Natl. Acad. Sci. U.S.A., 80 (1983) 2091-2094.

4 Dombrowski, A.M. and Kauffman, F.C., Growth of adult rat superior cervical ganglion explants in serum-free media, Brain Research, 219 (1981) 407-421. vious when the $1 \times$ objective was used. Although more pixels should improve growth estimates, the time required to compute the growth value would increase accordingly if equivalent computational power is used. It is doubtful that the improvement in accuracy would be sufficient to make up for the increased computational time required, especially when a typical experiment involves the measurement of hundreds of explants. Dedicated, commercial image processing systems also have the drawback that they cost several times more than our system (which can be easily constructed for less than $\$ 5000$, excluding the microscope) and still require extensive programming to perform tasks as specialized as neurite recognition and quantitation.

The system we have developed also has potential for other scientific applications. Aside from its use as a general image processor, we have found that with slight modifications it can be used as a video densitometer. As such, it has been successfully used to quantitate charred spots on thin layer chromatograms (Ford-Holevinski and Radin, in press) and may also prove useful in the analysis of autoradiograms. Software for the operation of this system and details of its construction are available by application to the authors.

\section{ACKNOWLEDGEMENTS}

The authors thank Dr. E. Delp for his assistance with the image processing algorithms used and $\mathrm{J}$. Haensley for the initial programming efforts. We also thank J. Hopkins for providing the explant cultures. This work was supported by NIH Grant EY 05947 and Training Grant MH 15794.

5 Ford-Holevinski, T.S., Radin, N.S. and Agranoff, B.W., Applications of a microcomputer-based video analyzer: neurite outgrowth rates and densitometry, Soc. Neurosci. Abstr., 8 (1982) 302.

6 Gayer, A. and Schwartz, M., The use of image analysis for measuring neuritic outgrowth from goldfish regenerating retinal explants, J. Neurosci. Meth., 7 (1983) 275-280.

7 Greene, L.A. and Shooter, E.M., The nerve growth factor: biochemistry, synthesis and mechanism of action, Annu. Rev. Neurosci., 3(1980) 353-402.

8 Heacock, A.M., Glycoprotein requirement for neurite outgrowth in goldfish retina explants: effects of tunicamycin, Brain Research, 241 (1982) 307-315.

9 Heacock, A.M. and Agranoff, B.W., Clockwise growth of 
neurites from retinal explants, Science, 198 (1977) 64-66.

10 Landreth, G.E. and Agranoff, B.W., Explant culture of adult goldfish retina: effect of prior optic nerve crush, Brain Research, 118 (1976) 299-303.

11 Landreth, G.E. and Agranoff, B.W., Explant culture of adult goldfish retina: A model for the study of CNS regeneration, Brain Research, 161 (1979) 39-53.

12 Mizel, S.B. and Bamburg, J.R., Studies on the action of nerve growth factor. I. Characterization of a simplified in vitro culture system for dorsal root and sympathetic ganglia, Dev. Biol., 49 (1976) 11-19.

13 Turner, J.E., Schwab, M.E. and Thoenen, H., Nerve growth factor stimulates neurite outgrowth from goldfish retinal explants: the influence of a prior lesion. Dev. Brain Res., 4 (1982) 59-66. 